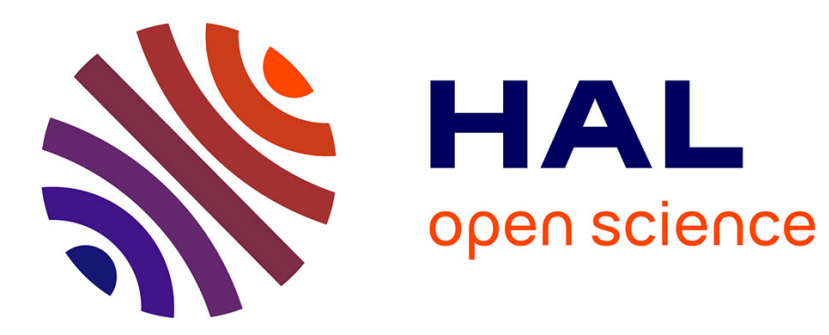

\title{
Multicolor two-photon light-sheet microscopy
}

Pierre Mahou, Julien Vermot, Emmanuel Beaurepaire, Willy Supatto

\section{To cite this version:}

Pierre Mahou, Julien Vermot, Emmanuel Beaurepaire, Willy Supatto. Multicolor two-photon lightsheet microscopy. Nature Methods, 2014, 11, pp.600-601. 10.1038/nmeth.2963 . hal-01048680

\section{HAL Id: hal-01048680 \\ https://hal-polytechnique.archives-ouvertes.fr/hal-01048680}

Submitted on 19 Nov 2014

HAL is a multi-disciplinary open access archive for the deposit and dissemination of scientific research documents, whether they are published or not. The documents may come from teaching and research institutions in France or abroad, or from public or private research centers.
L'archive ouverte pluridisciplinaire HAL, est destinée au dépôt et à la diffusion de documents scientifiques de niveau recherche, publiés ou non, émanant des établissements d'enseignement et de recherche français ou étrangers, des laboratoires publics ou privés. 


\title{
Multicolor two-photon light-sheet microscopy
}

\author{
Pierre Mahou ${ }^{1}$, Julien Vermot ${ }^{2}$, Emmanuel Beaurepaire ${ }^{1} \&$ Willy Supatto ${ }^{1}$ \\ (1) Laboratory for Optics and Biosciences, École Polytechnique, Centre National de la Recherche Scientifique (UMR 7645) and Institut National de la \\ Santé et de la Recherche Médicale (U696), Palaiseau, France. \\ (2) Institut de Génétique et de Biologie Moléculaire et Cellulaire, Centre National de la Recherche Scientifique (UMR 1704), Institut National de la Santé \\ et de la Recherche Médicale (U964) and Université de Strasbourg, Illkirch, France. \\ e-mail: emmanuel.beaurepaire@polytechnique.edu or willy.supatto@polytechnique.edu
}

Two-photon microscopy is the most effective approach for deep-tissue fluorescence cellular imaging; however, its application to high-throughput or high-content imaging is often hampered by low pixel rates, challenging multicolor excitation and potential cumulative photodamage. To overcome these limitations, we extended our prior work and combined two-photon scanned lightsheet illumination (or two-photon selective-plane illumination microscopy, 2P-SPIM) ${ }^{1}$ with mixed-wavelength excitation ${ }^{2}$ to achieve fast multicolor two-photon imaging with negligible photobleaching compared to conventional two-photon laser pointscanning microscopy (2P-LSM). We report on the implementation of this strategy and, to illustrate its potential, recorded sustained four-dimensional (4D: three dimensions + time) multicolor two-photon movies of the beating heart in zebrafish embryos at 28- $\mathrm{MHz}$ pixel rates.

To obtain multicolor two-photon excitation by wavelength mixing, we spatially and temporally overlapped two pulse trains produced by a femtosecond laser and an optical parametric oscillator (Fig. 1a). The two beams separately generate two-photonexcited fluorescence (2PEF; Fig. 1a) of blue and red chromophores, and their spatiotemporal overlap provides an additional twophoton excitation route to probe a third chromophore (i.e., in the green-yellow range) through twocolor two-photon-excited fluorescence2 (2C-2PEF; Fig. 1a). To implement this multicolor strategy, we added an optical parametric oscillator, a delay line and a spectral image splitter to a bidirectional 2P-SPIM setup (Fig. 1b, Supplementary Fig. 1 and Supplementary Methods). Implementation of wavelength mixing in the 2P-SPIM geometry differs from the 2P-LSM case in several ways, including the orthogonal configuration of excitation and detection axes, weakly focused excitation, bidirectional illumination and camera-based wide-field detection in 2P-SPIM (Supplementary Methods). We temporally synchronized the two pulse trains in both illumination paths by matching their chromatic dispersion (Supplementary Fig. 2). In turn, the reduced sensitivity to illumination chromatic aberration in 2P-SPIM enabled us to straightforwardly achieve spatial overlapping of the two beams and wavelength mixing over a larger field of view than is possible with 2P-LSM (Supplementary Fig. 3). Overall, we obtained efficient implementation of mixed-wavelength 2P-SPIM with bidirectional illumination, as illustrated by simultaneous trichromatic twophoton imaging of fly embryos (Fig. 1c).

Sustained two-photon excitation of multiple fluorescent proteins is limited by photobleaching: for instance, illumination in the 800-nm-wavelength range required for blue fluorophore excitation increases the photobleaching rate of red fluorescent proteins 3 (Supplementary Fig. 4). To compare the photobleaching induced by multicolor 2P-SPIM and 2P-LSM when using mixedwavelength excitation, we recorded images of live fly embryos with both microscopes using similar acquisition parameters (acquisition time, average power, signal level and spatial resolution; Supplementary Table 1). Multicolor 2P-SPIM induced substantially lower photobleaching than did 2P-LSM: we observed a 25-fold decrease for 2PEF of RFP and undetectable photobleaching for 2C-2PEF of GFP (Fig. 1d, Supplementary Fig. 4 and Supplementary Methods). When both methods are set up so as to obtain comparable fluorescence signal levels, reduced photobleaching in multicolor 2P-SPIM originates from lower peak intensities and different illumination dynamics relative to those of $2 \mathrm{P}-\mathrm{LSM}^{1,4}$. As a consequence, multicolor $2 \mathrm{P}$-SPIM allows the use of higher average powers to improve signal-to-noise ratio or increase imaging speed.

Finally, to demonstrate high-speed multicolor two-photon imaging, we recorded 3D time-lapse images of the beating heart in zebrafish embryos exhibiting CFP, GFP and DsRed labeling of pericardial, myocardial and red blood cells, respectively (Supplementary Methods and Supplementary Table 2). We acquired fast time series of multicolor two-photon images (up to 85 frames/s and 28 million pixels/s) at successive depths allowing 3D reconstruction of the heart periodic motion ${ }^{5}$ (Fig. 1e,f, Supplementary Video 1 and Supplementary Methods). Notably, multicolor 2P-SPIM imaging of the beating heart did not induce detectable photodamage (Supplementary Fig. 5) and provided 2C-2PEF signal with sufficient spatiotemporal resolution for quantitative 3D tracking of myocardial cells moving at up to $600 \mu \mathrm{m} / \mathrm{s}$ within the entire heart (Fig. 1g and Supplementary Video 2).

The combination of mixed-wavelength excitation with light-sheet illumination provides a robust and efficient way to achieve multicolor two-photon tissue imaging at high pixel rates and signal levels while dramatically reducing photobleaching. This strategy should prove invaluable in systems biology. 
Note: Any Supplementary Information and Source Data files are available in the online version of the paper (doi:10.1038/nmeth.2963).

\section{ACKNOWLEDGMENTS}

We thank A. McMahon and A. Stathopoulos (Caltech) for the $m c d 8-G F P / H 2 A R F P$ Drosophila line, J.M. Sintes and S. Roth for technical assistance, and T.V. Truong for discussions. We thank M. Liebling and the University of California at Santa Barbara for providing Cardiac Image-Sequence Synchronization Software. This work was supported by the French Agence Nationale de la Recherche (ANR-2010-JCJC-1510-01, ANR-09-PIRI-0013, ANR-11-EQPX-0029), European Union Seventh Framework Programme (Marie Curie International Reintegration Grant no. 268379), Fondation Louis D de l'Institut de France, Human Frontier Science Program and INSERM.

\section{AUTHOR CONTRIBUTIONS}

P.M., E.B. and W.S. conceived of the project and implemented the optical setup. P.M. and W.S. performed fly and zebrafish imaging and image analysis. J.V. provided and labeled the zebrafish lines. P.M., E.B. and W.S. wrote the manuscript.

\section{REFERENCES}

1. Truong, T.V., Supatto, W., Koos, D.S., Choi, J.M. \& Fraser, S.E. Nat. Methods 8, 757-760 (2011).

2. Mahou, P. et al. Nat. Methods 9, 815-818 (2012).

3. Andresen, V. et al. Curr. Opin. Biotechnol. 20, 54-62 (2009).

4. Mertz, J. Nat. Methods 8, 811-819 (2011).

5. Liebling, M., Forouhar, A.S., Gharib, M., Fraser, S.E. \& Dickinson, M.E. J. Biomed. Opt. 10, 054001 (2005).

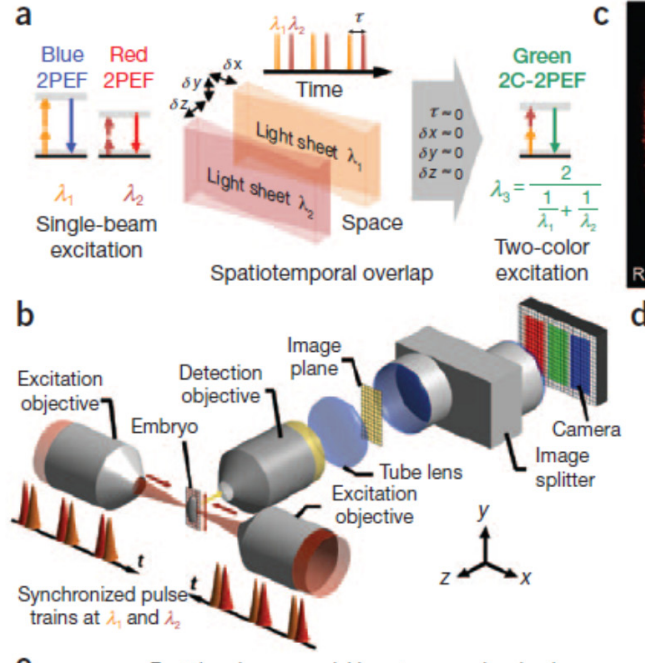

e Fast time-lapse acquisition at successive depths
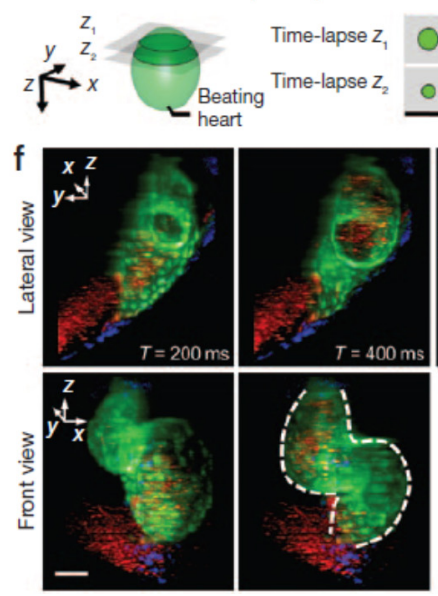

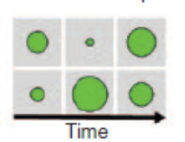

(1)
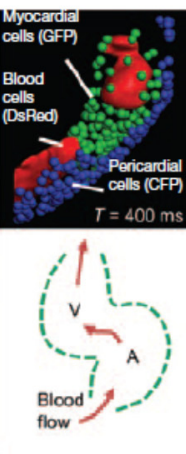

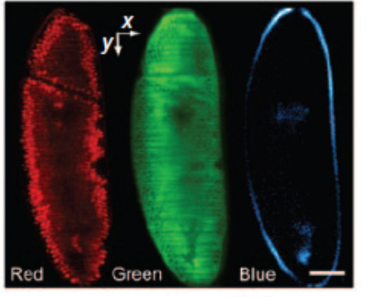

d RFP
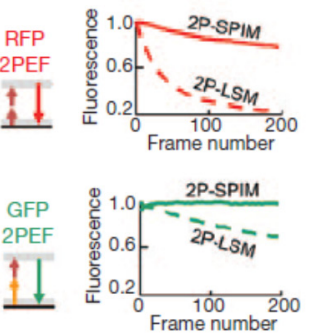

$3 \mathrm{D}+\mathrm{T}$ reconstruction
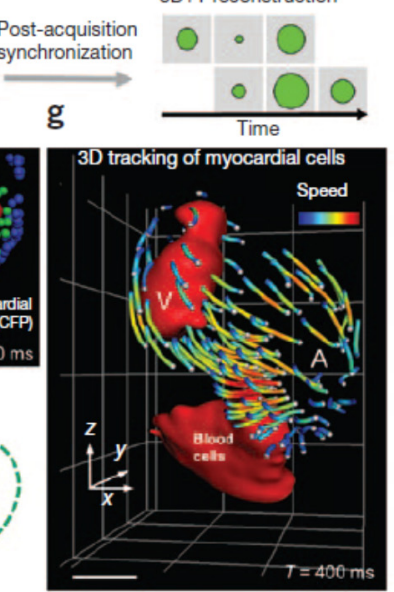

Figure 1 | Fast trichromatic two-photon imaging of live embryos using mixed-wavelength lightsheet excitation. (a) Principles of mixed wavelength excitation in 2P-SPIM geometry. (b) Optical setup of a multicolor 2P-SPIM microscope with bidirectional illumination. Two synchronized femtosecond pulse trains $(\lambda 1=$ $820-840 \mathrm{~nm}$ and $\lambda 2=1,090-1,175 \mathrm{~nm})$ are vertically scanned at the foci of two lownumerical aperture (NA) objectives to create bidirectional light-sheet illumination. The image is collected by an orthogonal detection objective, and spectral components are spatially separated on an electron-multiplying charge-coupled device (EMCCD) camera by an image splitter. (c) Simultaneous trichromatic $2 \mathrm{PEF}$ imaging of a GFP- and RFP-labeled gastrulating fly embryo showing endogenous signal from the yolk and the vitelline membrane (blue), green labeling of the cell membranes and red labeling of the nuclei. (d) Reduced photobleaching of RFP and GFP using 2P-SPIM compared to 2P-LSM with similar acquisition parameters (see Supplementary Fig. 4). (e-g) Fast multicolor 2P-SPIM imaging of a zebrafish embryo heart at $2 \mathrm{~d}$ post fertilization and exhibiting CFP, GFP and DsRed labeling of nuclei, myocardial cells and red blood cells, respectively. (e) 2D series are recorded at a rate of 48-85 frames/s in successive planes with no appreciable bleaching, and the periodic contractions are reconstructed in three dimensions using post-processing synchronization with typically $0.4-\mu \mathrm{m}$ lateral and $3-\mu \mathrm{m}$ axial optical resolution within a $210 \times$ $230 \times 300 \mu \mathrm{m} 3$ acquisition volume and $0.67 \times$ $0.67 \times 2.0 \mu \mathrm{m} 3$ voxel size (Supplementary Videos 1 and $\mathbf{2}$ and Supplementary Methods). (f,g) 2C-2PEF signal from GFP-labeled myocardial cells (f) is sufficient for 3D cell tracking (g). V, ventricle; A, atrium. Color-coded speed ranges from 0 to $620 \mu \mathrm{m} / \mathrm{s}$; grid spacing is $67 \mu \mathrm{m}$. Scale bars, $50 \mu \mathrm{m}$. 